\title{
Analysis of Novel Variable Reluctance Resolver with Asymmetric Teeth on the Stator
}

\author{
Chengjun Liu, Ming QI, and Meng Zhao \\ Department of Electrical Engineering and Automation, Harbin Institute of Technology, Harbin 150001, China
}

Correspondence should be addressed to Chengjun Liu; liucj@hit.edu.cn

Received 15 February 2013; Accepted 22 May 2013

Academic Editor: Dan Zhang

Copyright (C) 2013 Chengjun Liu et al. This is an open access article distributed under the Creative Commons Attribution License, which permits unrestricted use, distribution, and reproduction in any medium, provided the original work is properly cited.

The variable reluctance (VR) resolver is usually used to measure the shaft angle of motor. A novel VR resolver with asymmetric teeth on the stator is proposed to achieve the minimal number of active teeth and eliminate the amplitude imbalance of the output voltages of SIN and COS coils by bringing the fifth compensating tooth. The structure and the total output voltages of signal windings are explicated theoretically in detail. The topology of such a machine just requires 2D finite element analysis (FEA) to accurately predict the machine performance. Results of FEA and principle of VR resolver with asymmetric teeth are in good agreement, and several special relevant problems are studied. Finally, experimental results validate the theoretical analysis and FEA of the proposed VR resolver.

\section{Introduction}

When vector control, direct torque control, and other control strategies are applied in AC governor system with high dynamic performance, the rotor angular position is required necessarily [1-4]. As electromagnetic rotor position sensor of motor, variable reluctance (VR) resolver, which has simple structure, small axial dimension, high reliability, high amplitude of output voltage, low cost, and relatively high precision, is widely used to realize commutation and control algorithms in numerous applications especially in electric vehicle [5-10].

The conventional VR resolver adopts the sophisticated sinusoidal distributed windings placed on the stator and has slots in both stator and rotor to output two quadrature signals [11]. However, the turns of reference winding and signal windings should be an integral number, and thus the angle measurement accuracy will be reduced $[12,13]$. Therefore, the novel VR resolver has concentrated windings used for SIN and COS coils and rotor with several salient poles to induce voltages containing the information of the rotor angle [1416]. Several researches also focus on the resolver models and new-type resolver $[17,18]$.

Usually, for a conventional 4 pairs of pole VR resolver, the number of teeth on stator is selected as at least 8 or the integer multiple of 8. Unfortunately, when VR resolvers are applied in a small and narrow space; for example, when the stator diameter is less than $30 \mathrm{~mm}$, it is of great difficulty to insert coils because thin copper wires are too fragile and the space inside the resolver for inserting coils manually is too limited to accomplish accurately and conveniently. Thus, a novel VR resolver with asymmetric teeth on the stator, which has a minimal number of active teeth and the simplest structure, is proposed to reduce the size and cost of the device while simplifying its construction without loss of accuracy.

This paper is organized as follows. Firstly, the construction and work principle of novel VR resolver with asymmetric teeth on the stator are introduced in Section 2. The expressions of air-gap permeance and the output voltages of signal windings are deduced. Section 3 shows results of FEA to validate the analysis of novel VR resolver with asymmetric teeth. Related problems including the difference between SIN and COS compensating coils and arbitrariness of the location chosen for the compensating tooth are emphasized. The prototype and test bench are developed based on the analysis and computation results above; the performance of VR resolver with asymmetric teeth is investigated in Section 4, including the output voltages of signal windings and zero-error. Conclusions are summarized in Section 5. 


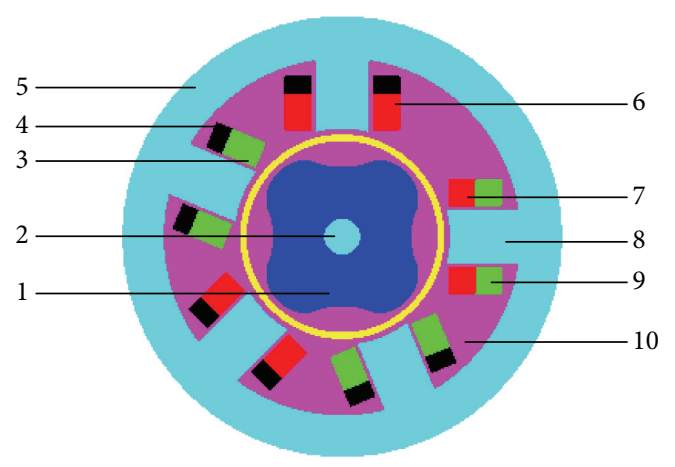

FIGURE 1: Structure of novel VR resolver with asymmetric teeth on the stator: 1-rotor, 2-shaft, 3-SIN coil, 4-reference winding, 5-stator, 6-COS coil, 7-COS compensating coil, 8-compensating tooth, 9-SIN compensating coil, 10-air.

\section{Principle of Novel VR Resolver with Asymmetric Teeth on the Stator}

2.1. Structure of VR Resolver with Asymmetric Teeth. The novel variable reluctance resolver, which is completely different from conventional multipole VR resolver, has reference winding and signal windings placed on the stator and several salient poles on the rotor. Thus, due to salient pole effects, with the rotation of the rotor, the inductance of signal windings varies, and the voltages of SIN and COS coils, which contain the information of the rotor angle, can be induced. When the rotor rotates one revolution, the period number of the output signals will be the same as the rotor salient pole number. Normally the higher the rotor pole number is, the more position accuracy the VR resolver can achieve. The stator pole number $z$ should be the times of four to form two symmetrical phases. Moreover, the reference coils are connected in series to form $z$ poles. The polarity of coils will be positive/negative alternatively. In order to make the teeth number be the minimum, four active teeth with reference windings are put on the stator. Another fifth asymmetric tooth, nonactive, is proposed to eliminate the average component from the induced voltage.

The SIN signal should be always in quadrature with COS signal, that is, the SIN winding and the COS winding must be mechanically displaced 90 electrical degrees from each other in the stator. And, the principle of slot-pole combination of VR resolver should be followed strictly. As a result, the number of teeth on the stator is selected as five; the rotor pole number $p$ is chosen as four and the angle-interval of the neighboring tooth $\alpha$ on the stator should be 67.5 mechanical degrees. The structure of novel VR resolver with asymmetric teeth on the stator is shown as Figure 1.

It can be seen that the reference windings and the signal windings are placed together in the stator, and the polarity of reference windings and SIN and COS coils are positive/negative alternatively. GCAACGGATCATCATGTAA The two concentrated coils, called compensating coils, are placed on the compensating tooth and, respectively, connected with SIN and COS coils in series. The stator is not complex and can be manufactured by simply using normal

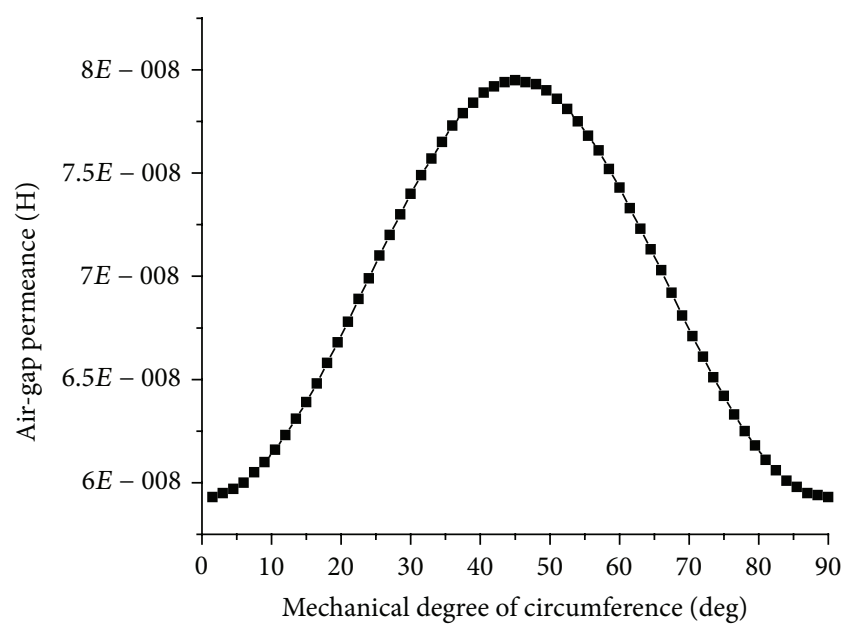

FIGURE 2: Waveform of air-gap permeance beneath the first tooth.

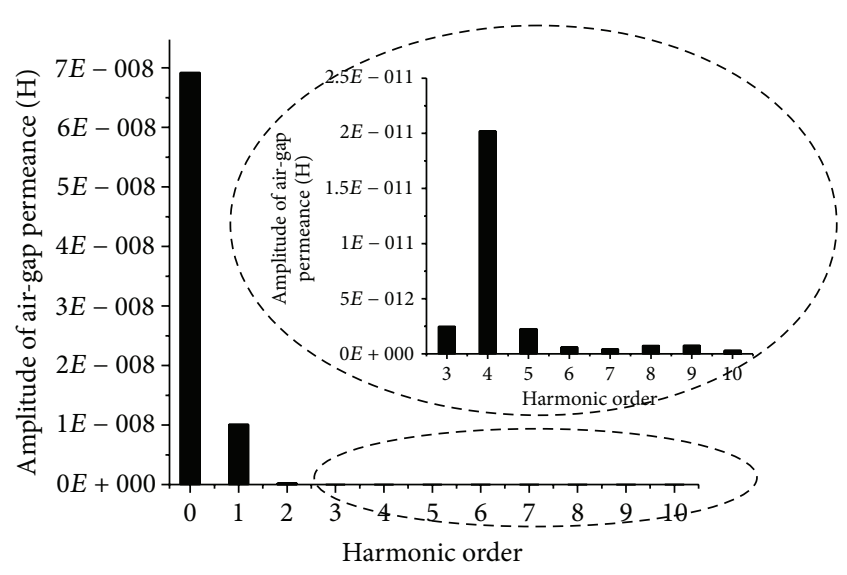

FIGURE 3: Harmonic components of air-gap permeance.

silicon steel and concentrated coils. To form the salient poles more precisely, the rotor may be a little more complicated to be fabricated by wire-electrode cutting. Thus, the novel VR resolver is named as VR resolver with asymmetric teeth for the reason that the angle-interval between the first four neighboring slots is 67.5 mechanical degrees and the fifth is 90 mechanical degrees. The shape of the compensating tooth is just like the other four teeth, and the main difference is that there is no reference winding on it.

\subsection{Analysis of the Air-Gap Permeance of VR Resolver with} Asymmetric Teeth. The air-gap permeance waveform determines the output voltage waveforms of SIN and COS coils directly. Invariably, the magnetic flux closes along the path where the permeance is the maximum. The main magnetic circuit contains stator yoke, stator teeth, rotor, and air-gap. So, analysis of the permeance under four active teeth should be taken as the principal thing.

According to the general analysis method for a VR resolver, the higher harmonics can be neglected [14]; thus, the 


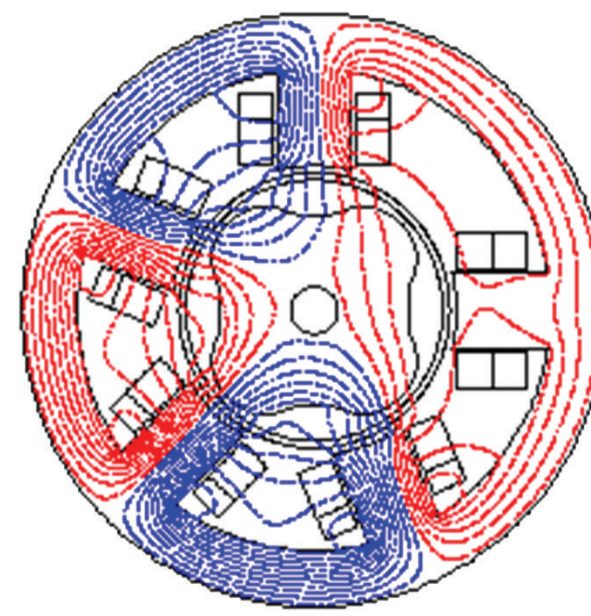

Isovalues results

Quantity: equi flux weber

Time (s): $2.5 E-6$ pos (deg): 0.015

Line/value

$1 /-31.13768 E-9$

$2 /-28.19886 E-9$

$3 /-25.26003 E-9$

$4 /-22.3212 E-9$

$5 /-19.38238 E-9$

$5 /-16.4435 E-9$
$7 /-13.50472 E-9$

$8 /-10.56589 E-9$

$9 /-7.62707 E-9$

/ $/ 7.62707 E-9$

$9 /-7.62707 E-9$
$11 /-1.74941 E-9$

$12 / 1.74941 E-9$

$12 / 1.18941 E-9$

$13 / 4.12824 E-9$
$14 / 7.06707 E-9$

$14 / 7.06707 E-9$
$15 / 10.0059 E-9$

$16 / 12.94472 E-9$

$17 / 15.88355 E-9$

$18 / 18.82238 E-9$

$19 / 21.76121 E-9$

FIgURE 4: Magnetic flux distribution in the VR resolver with asymmetric teeth on the stator.

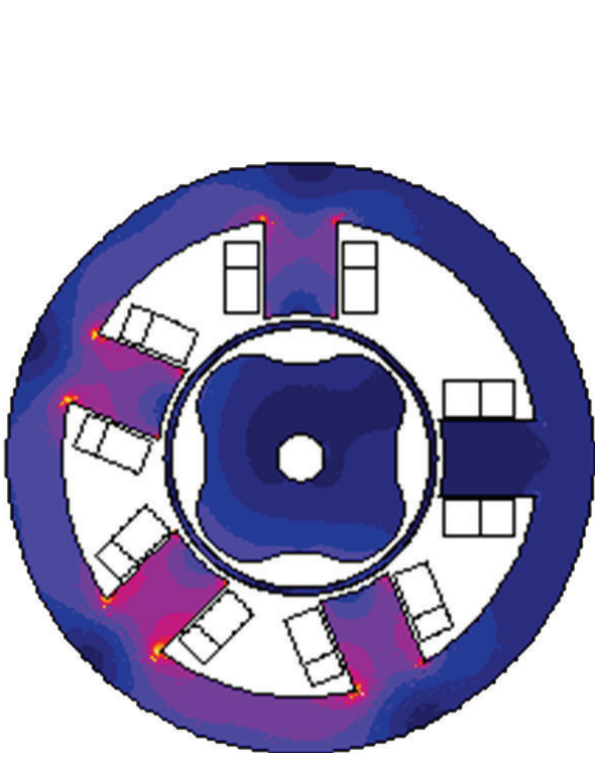

Color shade results
Quantity: |flux density| tesla
Time (s): $2.5 E-6$ pos (deg): 0.01
Scale/color
$327.77541 E-9 / 174.67212 E-6$
$174.67212 E-6 / 349.01651 E-6$
$349.01651 E-6 / 523.36085 E-6$
$523.36085 E-6 / 697.7052 E-6$
$697.7052 E-6 / 872.04954 E-6$
$872.04954 E-6 / 1.04639 E-3$
$1.04639 E-3 / 1.22074 E-3$
$1.22074 E-3 / 1.39508 E-3$
$1.39508 E-3 / 1.56943 E-3$
$1.56943 E-3 / 1.74377 E-3$
$1.74377 E-3 / 1.91812 E-3$
$1.91812 E-3 / 2.09246 E-3$
$2.09246 E-3 / 2.2668 E-3$
$2.2668 E-3 / 2.44115 E-3$
$2.44115 E-3 / 2.61549 E-3$
$2.61549 E-3 / 2.78984 E-3$

Figure 5: Magnetic flux density in the VR resolver with four teeth.

permeance versus rotor-position angle $\theta$ can be expressed as follows:

$$
\Lambda_{Z k}=\Lambda_{0}+\Lambda_{1} \cos p(\theta+k \cdot \alpha),
$$

where $\Lambda_{0}$ is the average permeance, $\Lambda_{1}$ is the amplitude of the fundamental component, $\theta$ is the rotating angle, $p$ is the salient pole pair number of the rotor, $\alpha$ is the mechanical degree of the neighboring tooth, and $k$ is the number of the stator teeth. For example, the number of the teeth at the top is 1 , and then other teeth are numbered by a counterclockwise rotation. as:

Thus, the sum of the air-gap permeance can be expressed

$$
\sum_{k=1}^{4} \Lambda_{Z k}=4 \Lambda_{0}+\sum_{k=0}^{3} \Lambda_{1} \cos p(\theta+k \cdot \alpha)=4 \Lambda_{0}
$$

Therefore, the sum of the air-gap permeance remains unchanged when the rotor rotates. Then, the reactance of the reference winding is a constant, and the amplitude of the induced voltage of reference winding which is excited by a constant voltage power supply is invariable so that no error will be brought in.

On the condition that reference winding is excited by a constant voltage power supply, the following equation should be followed when calculating the sum of the magneto motive force:

$$
\sum_{k=1}^{4} F_{z k}=\frac{\sum_{k=1}^{4} \Phi_{z k}}{\sum_{k=1}^{4} \Lambda_{z k}}=\mathrm{const}
$$

where $\sum_{k=1}^{4} \Phi_{z k}$ is the sum of the air-gap magnetic flux. 


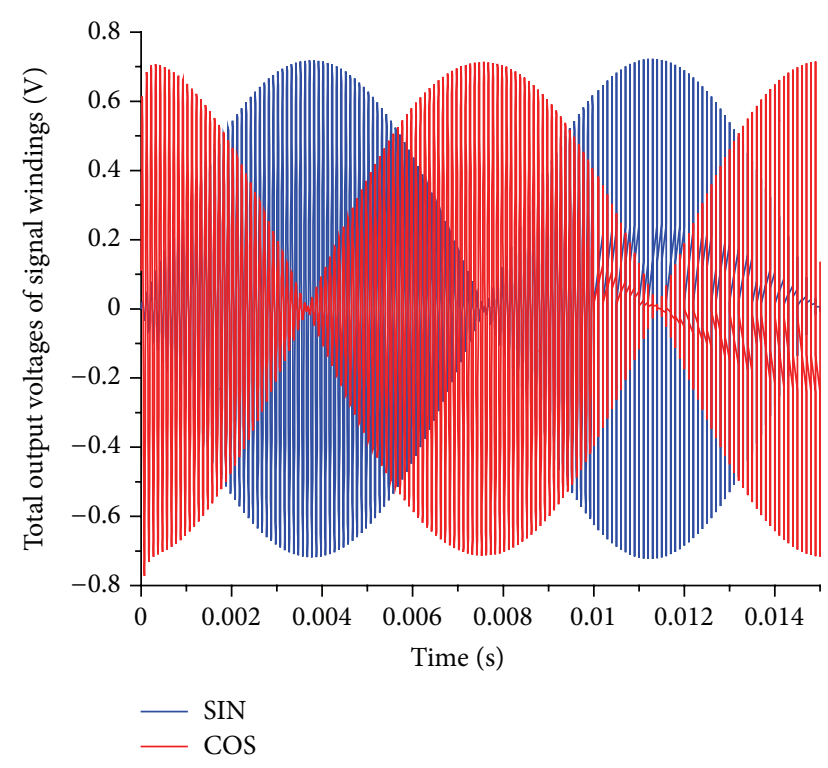

FIGURE 6: Total output voltages of signal windings.

Then, the air-gap magnetic flux of each tooth can be expressed as:

$$
\Phi_{z k}=F_{z k} \cdot \Lambda_{z k}=\Phi_{0}+\Phi_{1} \cos p(\theta+k \cdot \alpha),
$$

where $\Phi_{0}$ is the average magnetic flux and $\Phi_{1}$ is the amplitude of the fundamental component.

2.3. Analysis of the Output Voltages of SIN and COS Coils. To simplify the problem, it is assumed that

(1) the permeance of stator and rotor iron are infinite;

(2) the iron losses are omitted.

The output voltage of SIN coil will turn out to be

$$
E_{s}=\sum_{k=2 n}^{4} 4.44 f N_{s} \cos k \pi \Phi_{z k} \quad(n=1,2),
$$

where $N_{s}$ is the turns of each SIN coil, which is the concentrated coil like the reference winding, $f$ is the frequency of the reference signal, and $\cos k \pi$ represents the N/S polarity of reference coils.

Just like the air-gap permeance and the magnetic flux, the output voltage of SIN coil contains average component and fundamental component. The average component, which only varies with time and without the rotation of the rotor, is expressed as:

$$
E_{s 0}^{\prime}=\sum_{k=2 n}^{4} 4.44 f N_{s} \cos k \pi \Phi_{0}=0 .
$$

Nevertheless, the average value of signal windings cannot be neglected because of the following reasons.

(1) The reference winding and signal windings are put together on the stator tooth and magnetic coupling exists inevitably.
(2) There will be direct coupling between the windings due to the stray capacitance effect.

(3) The slot leakage flux will induce voltages irrelevant to the position signal.

(4) The magnetic circuit of VR resolver with asymmetric teeth is typically asymmetrical.

(5) The compensating effect of the signal windings in series will be little in that there exists only four active teeth.

Therefore, the average component of SIN coil should be revised as:

$$
E_{s 0}=Q_{s} f N_{s} \Phi_{0},
$$

where $Q_{s}$ is the voltage coefficient of SIN coil and can be calculated by FEA.

The fundamental component will turn out to be

$$
\begin{aligned}
E_{s 1} & =\sum_{k=2 n}^{4} 4.44 f N_{s} \Phi_{1} \cos k \pi \cos [p \theta+(k-1) \alpha] \\
& =8.88 f N_{S} \Phi_{1} \sin p \theta .
\end{aligned}
$$

Thus, the output voltage of SIN coil could be expressed in a more distinct way as:

$$
E_{s}=E_{s 0}+E_{s 1}=Q_{s} f N_{S} \Phi_{0}+8.88 f N_{S} \Phi_{1} \sin p \theta .
$$

Similarly, the output voltage of the COS coil will turn out to be

$$
E_{c}=E_{c 0}+E_{c 1}=Q_{c} f N_{c} \Phi_{0}+8.88 f N_{c} \Phi_{1} \cos p \theta,
$$

where $N_{c}$ is the turns of each concentrated COS coil and $Q_{c}$ is the voltage coefficient of $\mathrm{COS}$ coil and can also be calculated by FEA.

It can be clearly seen from (9) and (10) that the output voltages of VR resolver with asymmetric teeth contain average component and fundamental component. The average component will definitely cause function error and amplitude imbalance when the resolver measures the angle of the rotating shaft. Therefore, the output voltages of VR resolver with asymmetric teeth must be in series with compensating coils to eliminate the average value and obtain higher precision.

2.4. Analysis of the Total Output Voltages of Signal Windings. To simplify the issue, it is assumed that the magnetic flux in the air-gap under the compensating tooth is zero because there is no reference winding placed on it.

Then, based on the principle of continuity of magnetic flux, at any time, the algebraic sum of the magnetic flux in the compensating tooth and the other four active teeth should be zero.

Thus, the magnetic flux in the compensating tooth will turn out to be

$$
\Phi_{c}=-\sum_{k=1}^{4} \Phi_{z k}
$$




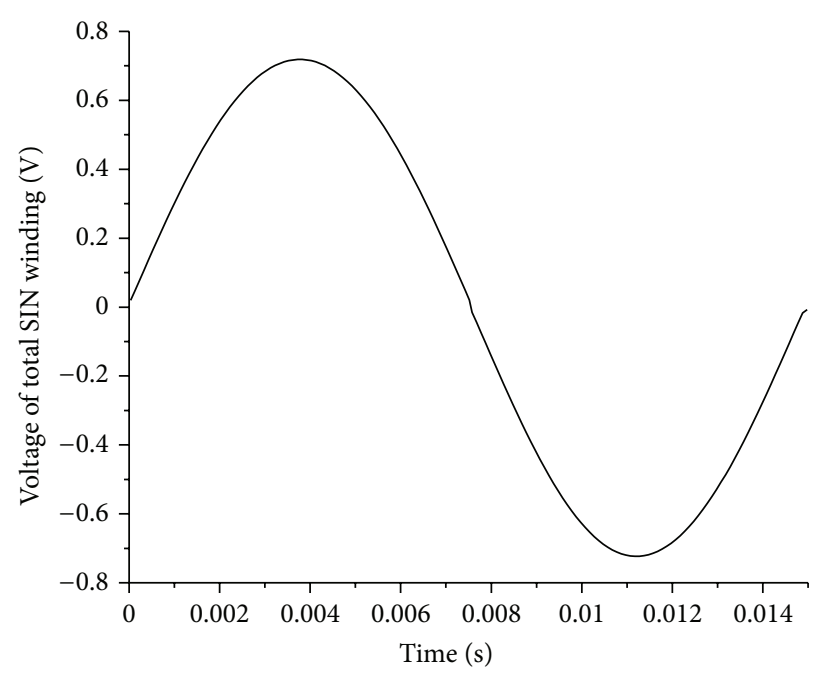

(a) Envelope curve

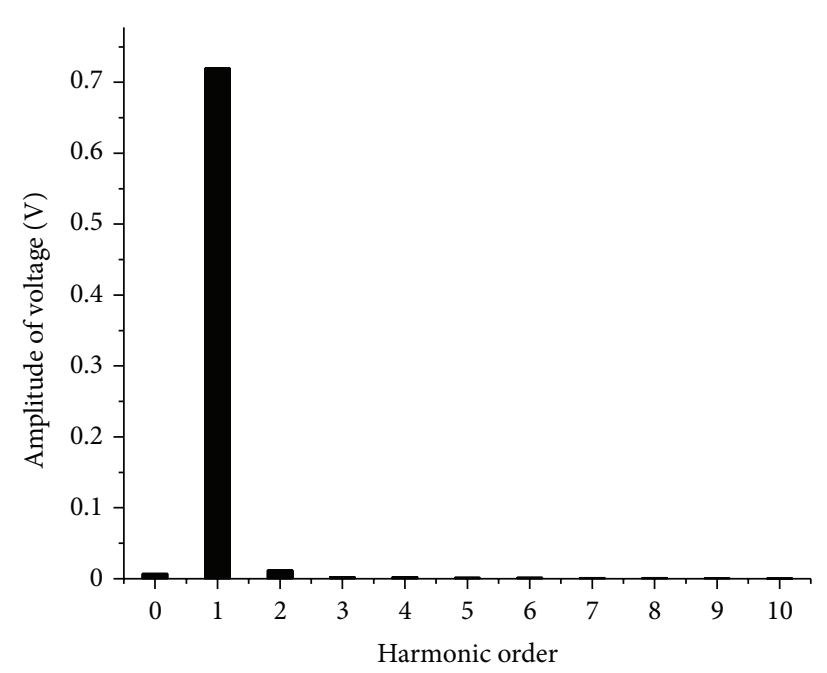

(b) Harmonic components

FIGURE 7: Voltage of total SIN winding.

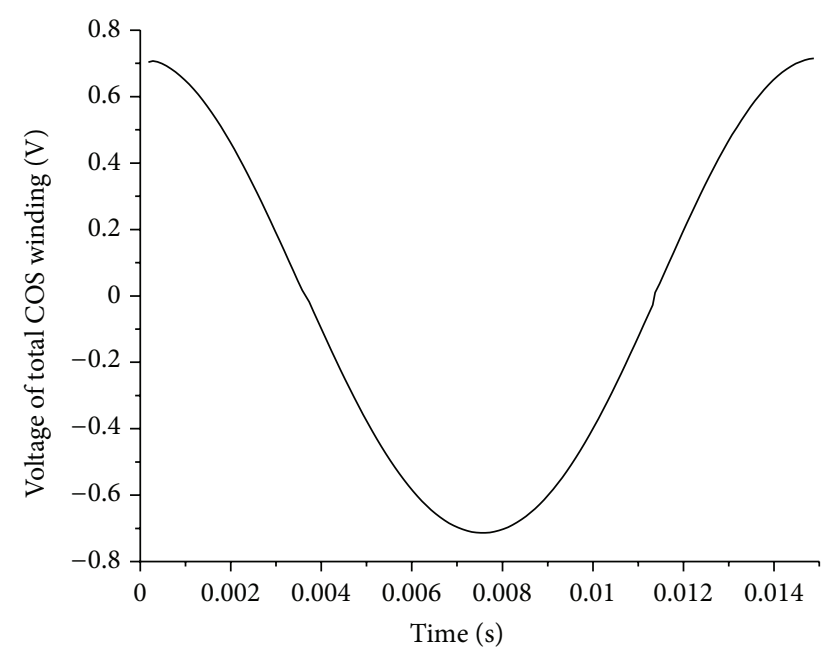

(a) Envelope curve

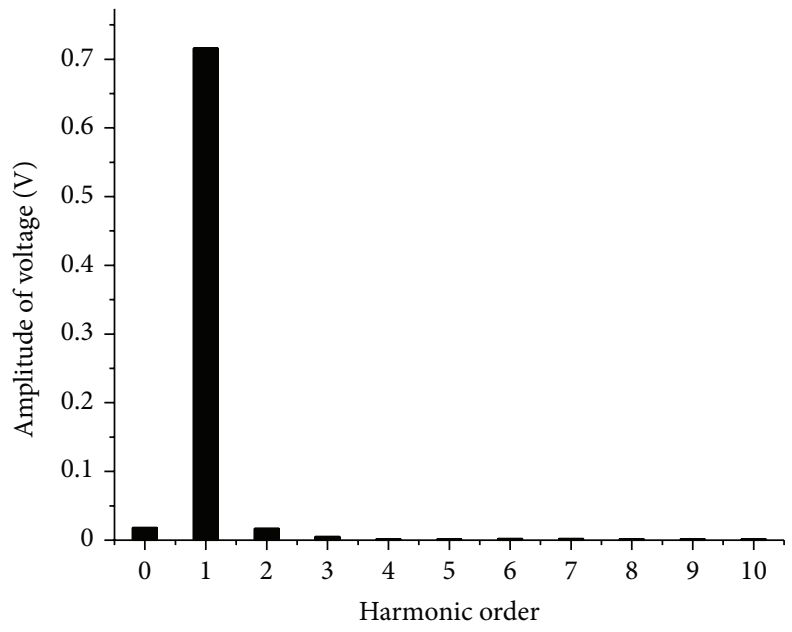

(b) Harmonic components

FIGURE 8: Voltage of total COS winding.

The average component of the output voltage of the SIN compensating coil can be expressed as:

$$
E_{c s 0}=-4.44 f N_{c s}\left(\sum_{k=1}^{4} \Phi_{0}\right)=-17.76 f N_{c s} \Phi_{0},
$$

where $N_{c s}$ is the turns of the SIN compensating coil.

The function of the compensating coils is to eliminate the average value from the induced voltages of SIN and COS coils, and the turns of compensating coils can only be calculated by FEA accurately.

The fundamental component of output voltage of the SIN compensating coil will turn out to be

$$
E_{c s 1}=-4.44 f N_{0} \sum_{k=1}^{4} \Phi_{1} \cos [4 \theta+(k-1) \alpha]=0 .
$$

Thus, by connecting the SIN coil with the SIN compensating coil in series, the total output voltage will be

$$
E_{s}^{\prime}=E_{s}+E_{c s}=8.88 f N_{s} \Phi_{1} \sin p \theta .
$$

Similarly, the total output voltages of the COS coil and the COS compensating coil will be

$$
E_{c}^{\prime}=E_{c}+E_{c c}=8.88 f N_{c} \Phi_{1} \cos p \theta .
$$

Obviously, after the compensating tooth and compensating coils are proposed, the total output voltages of the signal windings will only contain fundamental component and vary with the rotation of the rotor strictly, which can naturally obtain a higher precision to a great extent. This is the principle of novel VR resolver with asymmetric teeth on the stator. 


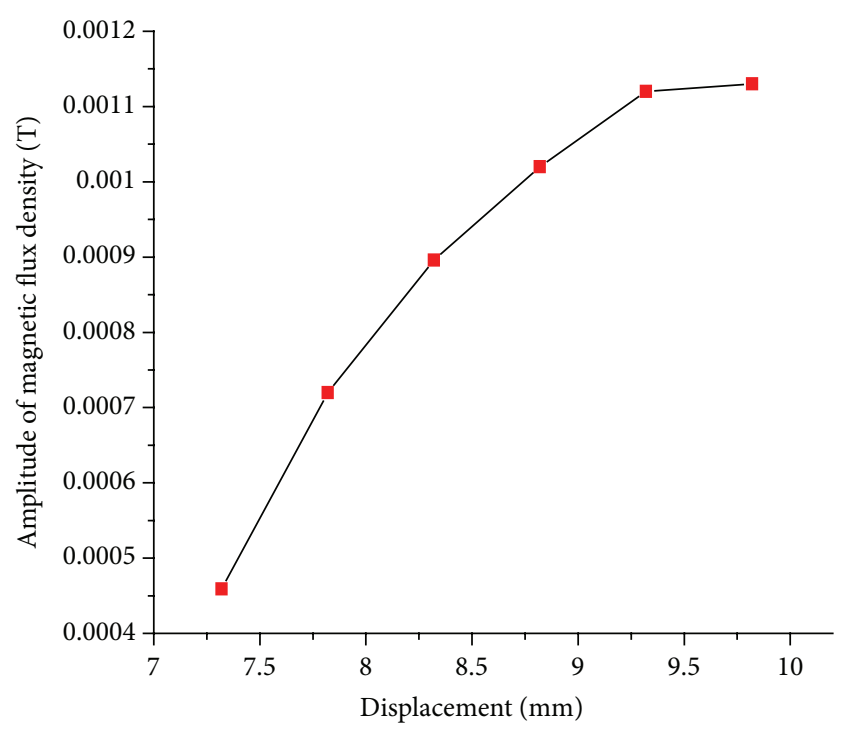

Figure 9: Magnetic flux density in the compensating tooth versus radial displacement along the symmetric line of compensating tooth.

TABLE 1: Specification of VR resolver with asymmetric teeth.

\begin{tabular}{lcc}
\hline Items & Units & Specification \\
\hline Exciting reference voltage & Vrms & 7 \\
Exciting frequency & $\mathrm{kHz}$ & 10 \\
Salient pole pair & $\mathrm{mm}$ & 4 \\
Minimum air-gap height & $\mathrm{mm}$ & 1 \\
Axial length & $\mathrm{mm}$ & 28 \\
Stator outer diameter & & 20 \\
Turns of each reference coil & & 20 \\
Turns of each signal coil & & 17 \\
Turns of the SIN compensating coil & & 42 \\
Turns of the COS compensating coil & & 0.072 \\
Voltage ratio & $\mathrm{rpm}$ & 1000 \\
Rotating speed & $\mu \mathrm{s}$ & 2.5 \\
Calculation time instant & & \\
\hline
\end{tabular}

\section{Finite Element Analysis}

FEA is used to simulate the performance of novel VR resolver with asymmetric teeth on the stator. The calculation time instant is related to exciting frequency and number of samples. The specification of novel VR resolver with asymmetric teeth is shown in Table 1.

3.1. Calculation of Air-Gap Permeance. The output voltages of signal windings depend on the air-gap permeance waveform. So, the calculation of air-gap permeance should be in the first place. Figure 2 shows the waveform of air-gap permeance beneath the first tooth. The harmonic components are shown in Figure 3, and the total harmonic distortion (THD) is 1.84\%. Results of the calculation of permeance validate the analysis of the air-gap permeance of VR resolver with asymmetric teeth on the stator.
TABLE 2: Calculated results of the total output voltages of signal windings.

\begin{tabular}{|c|c|c|}
\hline Items & Units & Specification \\
\hline $\begin{array}{l}\text { Maximum voltage value of total SIN } \\
\text { winding }\end{array}$ & $\mathrm{V}$ & 0.7186 \\
\hline Minimum voltage value of total SIN winding & $\mathrm{V}$ & -0.7232 \\
\hline Average value of total SIN winding & $\mathrm{V}$ & 0.0072 \\
\hline $\begin{array}{l}\text { Maximum voltage value of total COS } \\
\text { winding }\end{array}$ & $\mathrm{V}$ & 0.7154 \\
\hline $\begin{array}{l}\text { Minimum voltage value of total COS } \\
\text { winding }\end{array}$ & $\mathrm{V}$ & -0.7136 \\
\hline Average value of total COS winding & $\mathrm{V}$ & 0.0182 \\
\hline Electrical error & Degree & 0.6 \\
\hline
\end{tabular}

3.2. Calculation of the Total Output Voltages of Signal Windings. Figure 4 presents the magnetic flux distribution in the VR resolver with asymmetric teeth at $2.5 \mu$ s. It can be clearly seen from Figure 4 that the main magnetic circuit is asymmetrical, which will induce null voltages irrelevant to the position signal in the output windings to some extent. There exists a little amount of the slot leakage flux compared with the main flux and no magnetic flux under the compensating tooth. The magnetic flux density is shown in Figure 5, and the flux density is relatively low, which will not cause saturation in the stator and rotor. The magnetic flux density in the compensating tooth is lower than the other four teeth as a result of no reference winding on it.

The total output voltages of signal windings are almost equal between the positive and negative half of voltage cycle, as presented in Figure 6. After bringing the compensating tooth, the average value and amplitude imbalance can be reduced significantly and the precision will be improved effectively. Figure 7 shows the envelope curve and harmonic components of total voltage of SIN winding. Figure 8 displays the envelope curve and harmonic components of total voltage of COS winding.

Calculated results of the total output voltages of signal windings are shown in Table 2. Total SIN winding here includes SIN coil and SIN compensating coil and so does total COS winding.

It can be seen from Table 2 that the average value of voltage of total SIN winding and that of total COS winding are different because the turns of SIN and COS compensating coils are not equal. It should be noted that the average components can never be zero because the turns of the compensating coils should be an integral number. The explanation for the reason why SIN and COS compensating coils are not equal will be demonstrated in Section 3.3.

\subsection{Explanation for the Difference between SIN and COS} Compensating Coils. The turns of SIN compensating coil equal 17 and that of COS compensating coil are 42 , as shown in Table 1. Figure 9 presents the magnetic flux density in the compensating tooth versus radial displacement along the symmetric line of compensating tooth. It is supposed that the magnetic flux in the air gap under the compensating tooth 


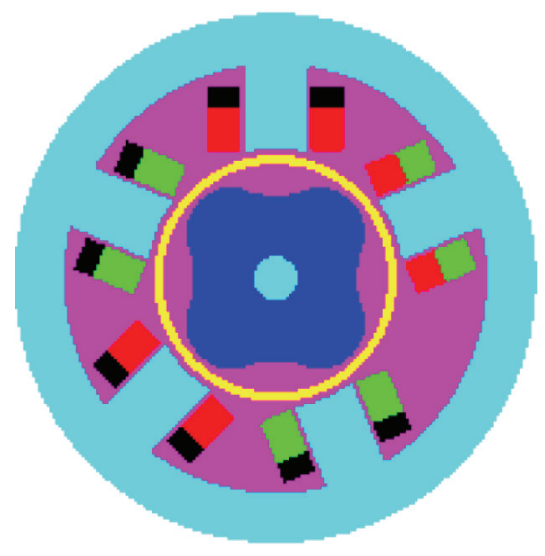

(a) Structure

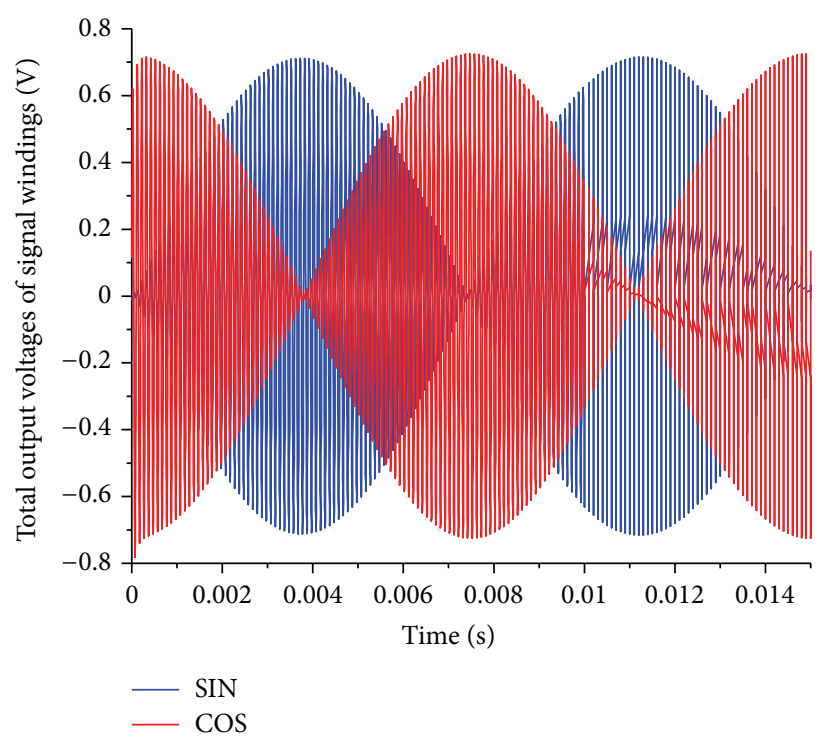

(b) Output voltages of signal windings

FIGURE 10: VR resolver which the angle-interval between the fourth tooth and compensating tooth is 90 mechanical degrees.

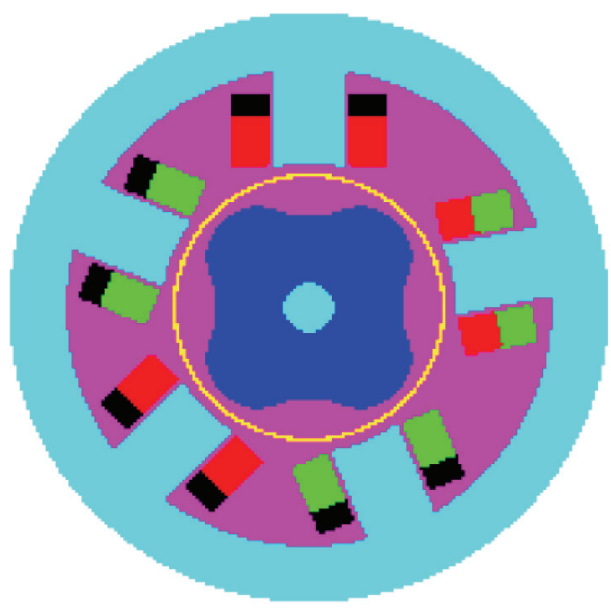

(a) Structure

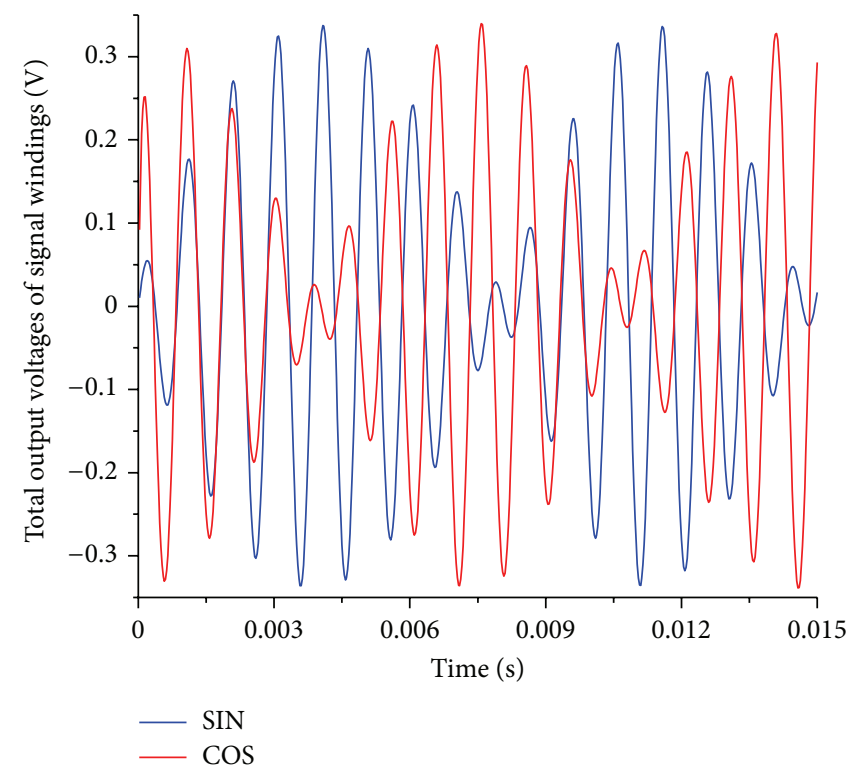

(b) Output voltages of signal windings

FIGURE 11: VR resolver which the angle-interval between the fourth tooth and compensating tooth is 78.75 mechanical degrees.

should be zero. The magnetic flux density in the compensating tooth varies with displacement along the symmetric line of compensating tooth. The amplitude of magnetic flux density will increase when the radial displacement is closer to the stator yoke. The turns of COS compensating coil is more than that of SIN compensating coil because COS compensating coil is farther away from the stator yoke.

3.4. Arbitrariness of the Location Chosen for the Compensating Tooth. Based on the principle of continuity of magnetic flux, expression (11) shows that the magnetic flux in the compensating tooth is irrelevant to the location of the fifth tooth. Therefore, the location chosen for the compensating tooth is arbitrary. Two typical examples are researched to validate the analysis above. Figure 10 shows the construction and output voltages of signal windings of one example which the angleinterval between the fourth tooth and compensating tooth is 90 mechanical degrees. The structure and output voltages of signal windings of another example which the angle-interval between the fourth tooth and fifth tooth is 78.75 mechanical degrees are shown in Figure 11. 


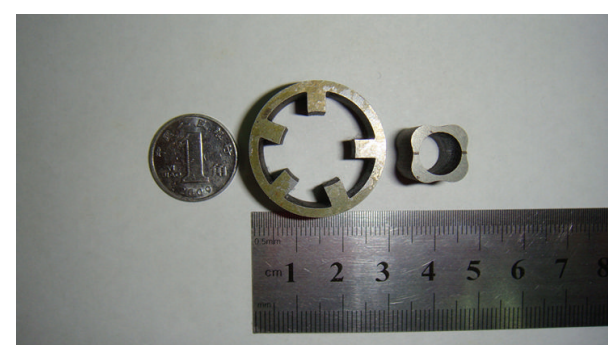

FIGURE 12: Prototype of novel VR resolver with asymmetric teeth on the stator.

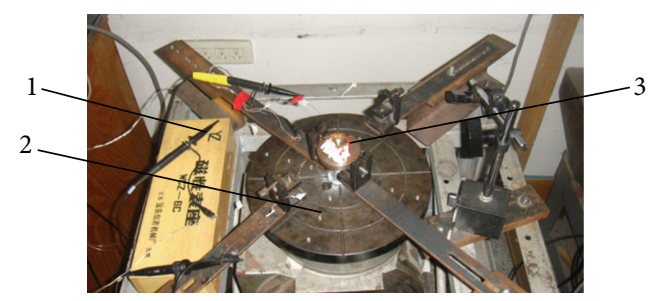

FIGURE 13: Experiment setup: 1-oscilloscope probe, 2-turntable, 3prototype.

Both two calculation examples are able to output two quadrature and amplitude-equal signals. The only difference between the three resolvers is that the turns of SIN and COS compensating coil are not invariable because of the distinctions of slot leakage flux distribution and the magnetic flux density in the compensating tooth.

\section{Experiment and Error Analysis}

4.1. Prototype. Based on the analysis and computations above, a prototype has been made as shown in Figure 12, with four active teeth and one nonactive tooth on the stator and four salient poles on the rotor.

\subsection{Output Voltages of Signal Windings Measurement.} Figure 13 presents the test bench for output voltages of signal windings measurement. The stator was fixed on the support, and the rotor was rotating with the shaft fixed on the turntable, and thus their relative positions can be changed. The output voltages of signal windings are measured by TEK oscilloscope, as shown in Figure 14. The experimental results prove the validities of the analysis and results of FEA above.

4.3. Zero-Error Measurement. Figure 15 shows the zero-error within a mechanical cycle which is measured by highprecision digital grating optical dividing head.

It can be seen that the maximum zero-error of SIN winding is $\pm 0.66^{\circ}$ and that of COS winding is $\pm 0.71^{\circ}$. The zero-error changes in a zigzag fashion at different zero positions because noneffective voltages exist in output signals. Compared with electrical error computed by FEA, results of the experiment is larger for the reason that it is difficult to make the stator and rotor absolutely concentric in the experiment.

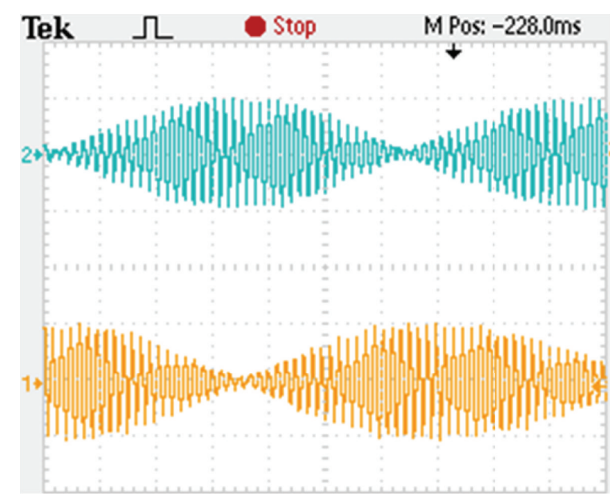

FIGURE 14: Oscilloscope graph measured: 1-voltage of COS winding, 2 -voltage of SIN winding.

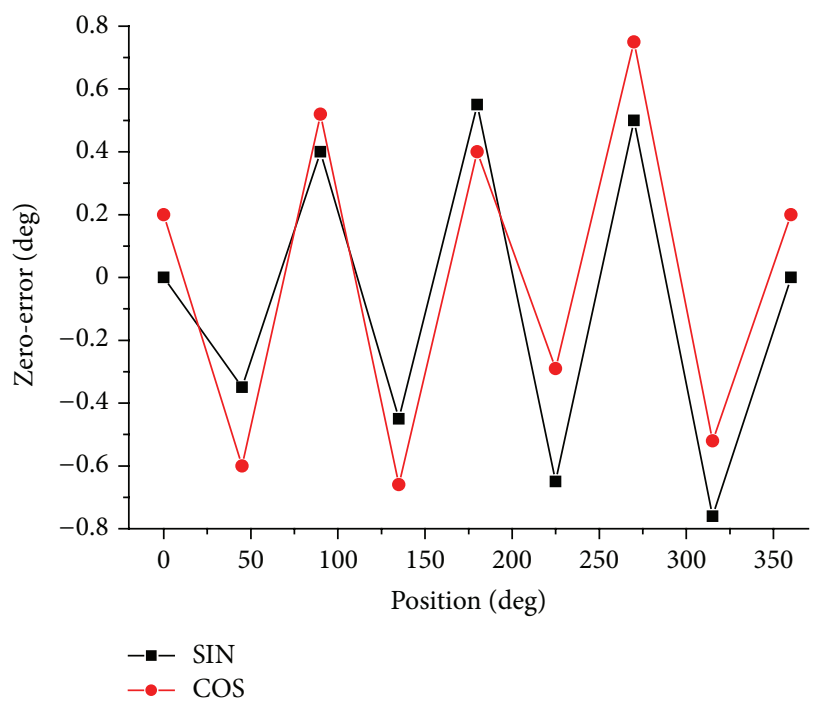

FIGURE 15: Zero-error versus rotating angle.

\section{Conclusions}

The following conclusions can be drawn from the analysis of the novel VR resolver with asymmetric teeth on the stator.

(1) The fifth asymmetric tooth, nonactive, called compensating tooth, plays a pivotal role in the new topology of VR resolver. The induced voltages of compensating coils can eliminate the average value from the voltages of SIN and COS coils so that the precision will be improved substantially.

(2) The calculated electric error of the proposed VR resolver can approach $\pm 0.7^{\circ}$. Several relevant special issues caused by special construction are researched in detail. Computations of FEA are consistent with the principle analysis of the proposed VR resolver.

(3) Experimental results show that the novel VR resolver has equivalent precision $\left( \pm 0.5^{\circ}\right)$ compared with the same type of conventional top VR resolvers. However, it is confirmed that the novel VR resolver with asymmetric teeth on the stator is advantaged with 
reliability and cost due to the simple manufacturing process and structure of stator. It is more cost effective, so, as position sensor, it is very useful for low cost drives or electric vehicles.

\section{Conflict of Interests}

The authors have stated explicitly that there is no conflict of interests in connection with this paper.

\section{Acknowledgments}

This work was supported by National Natural Science Foundation of China (51107013), Natural Scientific Research Innovation Foundation of Harbin Institute of Technology (HIT.NSRIF.2011141), and 973 Program under Grant No. 2013 CB035605.

\section{References}

[1] G. Pellegrino, R. I. Bojoi, and P. Guglielmi, "Unified directflux vector control for AC motor drives," IEEE Transactions on Industry Applications, vol. 47, no. 5, pp. 2093-2102, 2011.

[2] C. B. Jacobina, E. R. C. da Silva, and A. M. N. Lima, "Vector control strategies for single-phase induction motor drive systems," IEEE Transactions on Industrial Electronics, vol. 51, no. 5, pp. 1073-1080, 2004.

[3] S. Kuo-Kai, L. Jun-Kuh, and P. Van-Truong, "Global minimum torque ripple design for direct torque control of induction motor drives," IEEE Transactions on Industrial Electronics, vol. 57, no. 9, pp. 3148-3156, 2010.

[4] C. Patel, P. P. Rajeevan, A. Dey, R. Ramchand, K. Gopakumar, and M. P. Kazmierkowski, "Fast direct torque control of an open-end induction motor drive using 12-sided polygonal voltage space vectors," IEEE Transactions on Power Electronics, vol. 27, no. 1, pp. 400-410, 2012.

[5] K.-C. Kim, C. S. Jin, and J. Lee, "Magnetic shield design between interior permanent magnet synchronous motor and sensor for hybrid electric vehicle," IEEE Transactions on Magnetics, vol. 45, no. 6, pp. 2835-2838, 2009.

[6] K.-C. Kim, S.-J. Hwang, K.-Y. Sung, and Y. Kim, "A study on the fault diagnosis analysis of variable reluctance resolver for electric vehicle," in Proceedings of the 9th IEEE Sensors Conference (SENSORS '10), pp. 290-295, November 2010.

[7] L. Shao, Z. Tang, K. Maki, H. Funato, J. Moore, and G. Saikalis, "Integrated simulation and analysis of resolver sub-system for HEV electric drive," in Proceedings of the IEEE Vehicle Power and Propulsion Conference (VPPC '08), pp. 1-5, September 2008.

[8] A. Bünte and S. Beineke, "High-Performance speed measurement by suppression of systematic resolver and encoder errors," IEEE Transactions on Industrial Electronics, vol. 51, no. 1, pp. 4953, 2004.

[9] M. Konghirun, "A resolver-based vector control drive of permanent magnet synchronous motor on a fixed-point digital signal processor," in Proceedings of the IEEE Region 10 Conference: Analog and Digital Techniques in Electrical Engineering, pp. D167-D170, November 2004.

[10] A. Murray, B. Hare, and A. Hirao, "Resolver position sensing system with integrated fault detection for automotive applications," in Proceedings of the 1st IEEE International Conference on Sensors (IEEE Sensors '02), pp. 864-869, June 2002.
[11] D. C. Hanselman, R. E. Thibodeau, and D. J. Smith, "Variablereluctance resolver design guidelines," in Proceedings of the 15th Annual Conference of IEEE Industrial Electronics Society (IECON '89), pp. 203-208, November 1989.

[12] L. Knox, "Synchro and resolver performance definitions," IRE Transactions on Component Parts, vol. 3, no. 3, pp. 88-98.

[13] D. C. Hanselman, "Resolver signal requirements for high accuracy resolver-to-digital conversion," IEEE Transactions on Industrial Electronics, vol. 37, no. 6, pp. 556-561, 1990.

[14] L. Sun and Y. Lu, "Rotor-position sensing system based on one type of variable-reluctance resolver," in Proceedings of the 32nd Annual Conference on IEEE Industrial Electronics (IECON '06), pp. 1162-1165, November 2006.

[15] L. Sun, "Analysis and improvement on the structure of variable reluctance resolvers," IEEE Transactions on Magnetics, vol. 44, no. 8, pp. 2002-2008, 2008.

[16] L. Sun, J. Shang, and J. Zou, "New absolute rotor-position sensors for inverter-driven motors," in Proceedings of the Digests of the IEEE International Magnetics Conference (INTERMAG ASIA '05), pp. 975-976, Nagoya, Japan, April 2005.

[17] J. Figueiredo, "Resolver models for manufacturing," IEEE Transactions on Industrial Electronics, vol. 58, no. 8, pp. 3693-3700, 2011.

[18] F. Kimura, M. Gondo, A. Yamamoto, and T. Higuchi, "Resolver compatible capacitive rotary position sensor," in Proceedings of the 35th Annual Conference of the IEEE Industrial Electronics Society (IECON '09), pp. 1923-1928, November 2009. 


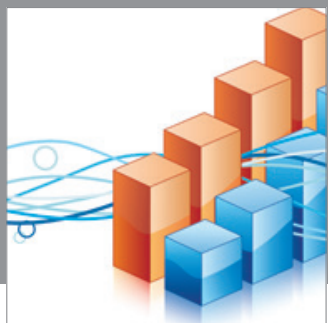

Advances in

Operations Research

mansans

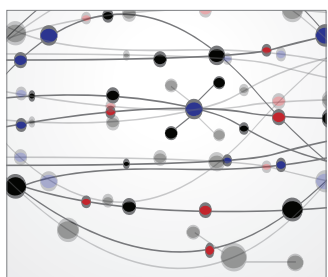

The Scientific World Journal
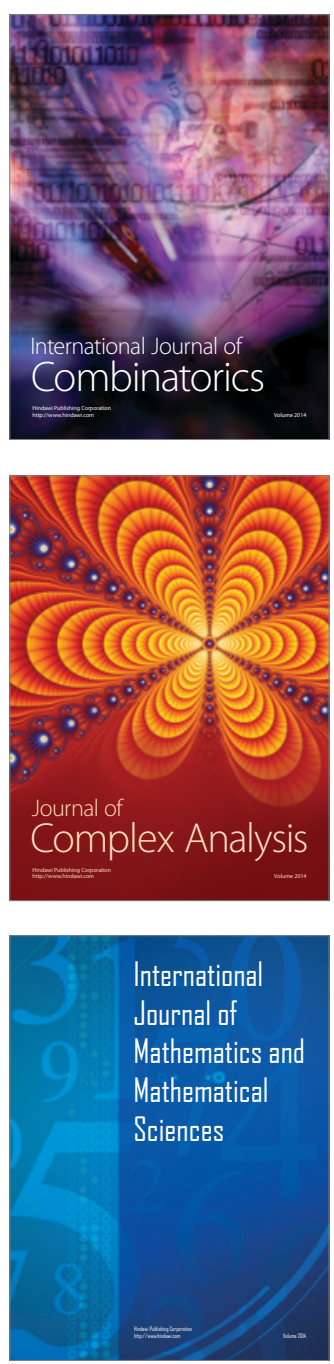
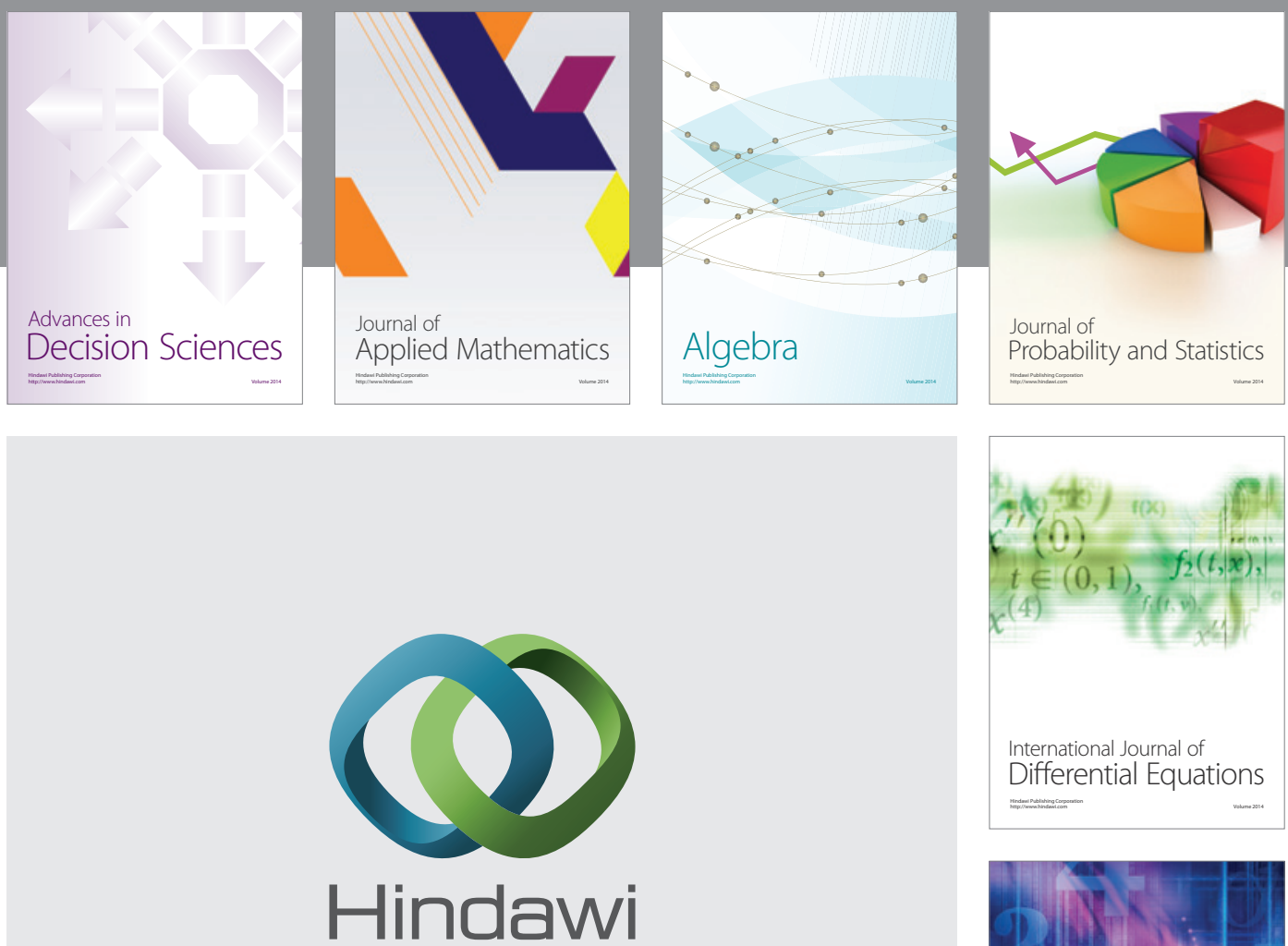

Submit your manuscripts at http://www.hindawi.com
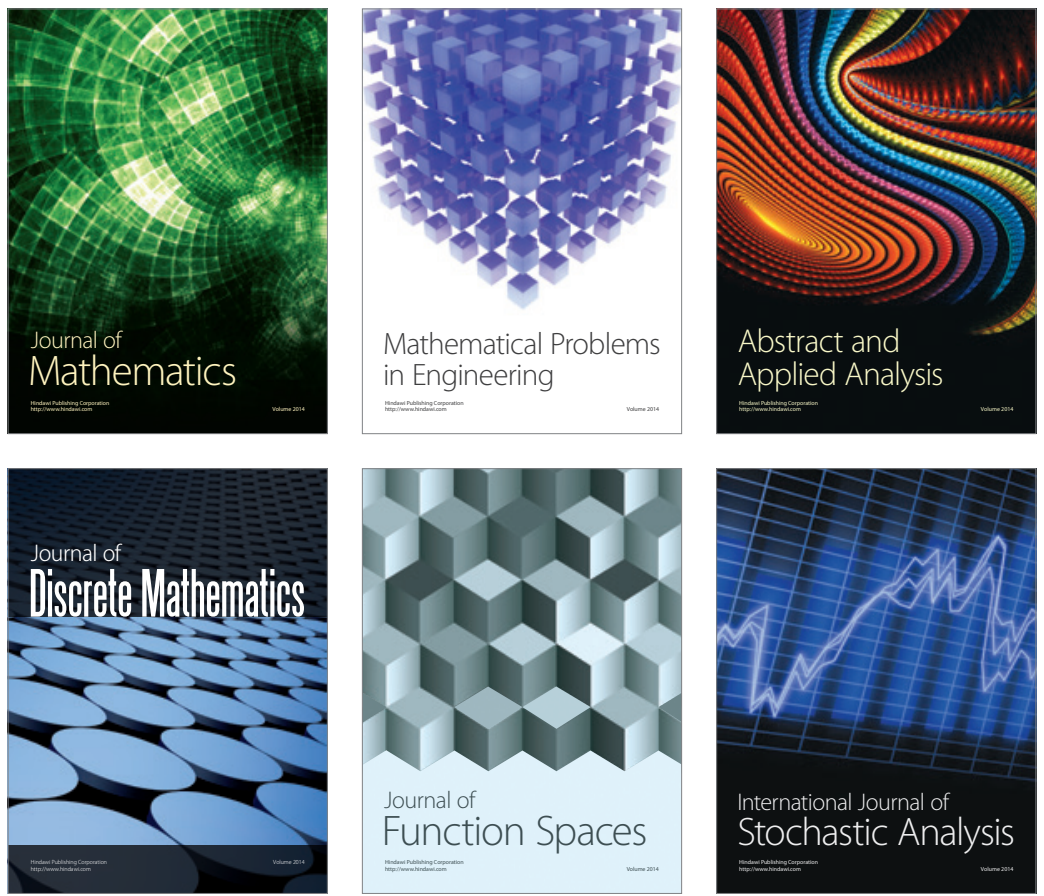

Journal of

Function Spaces

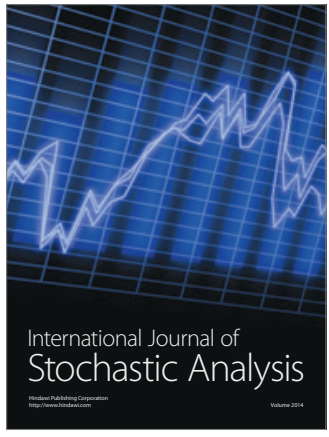

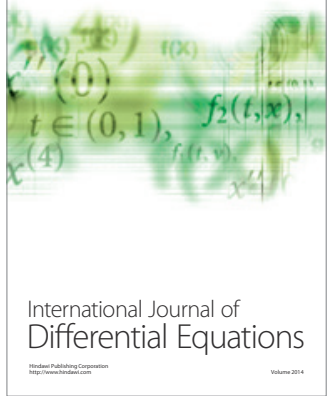
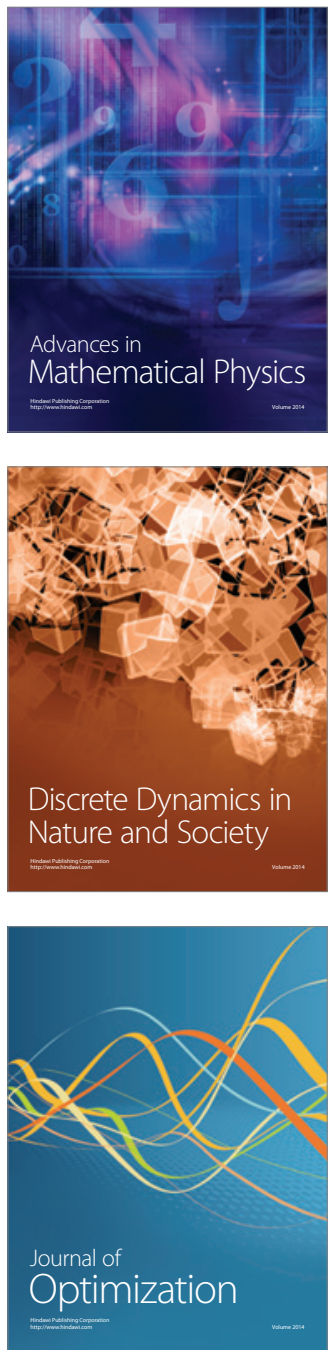